\title{
An Approach to Quantum Gravitodynamics and Some Important Results
}

\author{
Bakul Kumar Chakravorti*, Md Sakibul Islam, Syed Badiuzzaman Faruque
}

Department of Physics, Shahjalal University of Science and Technology, Sylhet, Bangladesh

\section{Email address:}

bkcb59@gmail.com (B. K. Chakravorti), sakibulislamshishir@gmail.com (Md S. Islam), awsbf62@yahoo.com (S. B. Faruque)

${ }^{*}$ Corresponding author

\section{To cite this article:}

Bakul Kumar Chakravorti, Md Sakibul Islam, Syed Badiuzzaman Faruque. An Approach to Quantum Gravitodynamics and Some Important Results. World Journal of Applied Physics. Vol. 5, No. 4, 2020, pp. 43-48. doi: 10.11648/j.wjap.20200504.11

Received: November 15, 2020; Accepted: December 1, 2020; Published: December 8, 2020

\begin{abstract}
We present in this article a new approach to the theory of gravitation. Here, the gravitational field of a gravitating body is assumed to be four fold. The gravitating body of mass $M$ possesses gravitoelectric mass $M$, gravitomagnetic mass $-2 M$. The test particle possess electric and magnetic masses that are algebraically of the same sign of that of the source body, i.e., $m$ and $-2 \mathrm{~m}$. Based on the electrostatic force formula, we find four forces acting on the test particle. The sum of these forces gives exactly the Newtonian gravitation force and gravitational potential energy. The classical theory of this approach yields four sets of Maxwellian equations and thus, four spin 1 bosons convey the complete force of gravity. The quantum version of this approach to gravity is illustrated here by presenting the fundamental Feynman diagrams that issue from the new theory. We work out scattering cross-section of interaction of an electron with a fixed gravitating mass partially. Of the four Feynman diagrams, we work out cross-section of scattering for only the simplest diagram. The non-relativistic limit of the cross-section is found and compared with that of electric Rutherford scattering. It is found that the electromagnetic cross-section is $10^{17}$ times larger than the gravitational cross-section for the one process we considered. The work is fundamental and sheds new light onto quantum gravitodynamics.
\end{abstract}

Keywords: Gravitodynamics, Gravitoelectromagnetigm, Newtonian Gravity, Feynman Diagram, Scattering Cross-section

\section{Introduction}

Linearized gravity stands in between Newtonian gravity and general relativity $[1,2]$. Linearized gravity leads a theory of gravity which is parallel to electromagnetism. Thus, we get Gravitoelectromagnetism [3]. In this theory, the source of gravity field and the test body are assumed to have opposite masses [4]. And a gravitomagnetic mass (or charge) is attributed to both the source and the test mass. Thus, a body of mass $M$ has gravitoelectric charge $M$ and a gravitomagnetic charge $2 M$, if the body is the source of gravitational field. On the other hand, for a test particle of mass $m$, the gravitoelectric charge is $(-m)$ and the gravitomagnetic charge $(-2 m)$. This prescription is adopted to ensure that gravity is always attractive [3, 4] Now, we all know that there are no evidences in favor of differences between source and test bodies regarding their that property which is called mass.

To avoid the seemingly justified, but conceptually unbearable convention of the opposite changes for source and test body, it has been proposed by Faruque [5] that the source body and test body should be treated alike. Therefore, it is conjectured [5] that a source of gravity of ordinary mass $M$ has gravitoelectric charge $q_{E}=M$ and a gravitomagnetic charge $q_{B}=-2 M$. In the same fashion, a test body of mass $\mathrm{m}$ has gravitoelectric charge $q_{E}=m$ and gravitomagnetic charge $q_{B}=-2 m$. It is also conjectured that the source body produce four fields:

$$
\begin{aligned}
\vec{E}_{e e} & =\frac{G M}{r^{2}} \hat{r}, \\
\vec{E}_{b b} & =-\frac{2 G M}{r^{2}} \hat{r}, \\
\vec{E}_{e b} & =\frac{3}{2} \frac{G M}{r^{2}} \hat{r}, \\
\vec{E}_{b e} & =-\frac{3 G M}{r^{2}} \hat{r} .
\end{aligned}
$$


Hence, it is assumed that $\vec{E}_{e e}$ has the electric charge as its source and it acts on the electric charge of the test particle. Similar concept applies to $\vec{E}_{b b}$. For $\vec{E}_{e b}$, its source is electric charge and it acts on magnetic charge. The force is given by the simple electrostatic formula [5]

$$
\vec{F}=q \vec{E}
$$

Hence, $\vec{F}_{1}=(m) \vec{E}_{e e}, \vec{F}_{2}=(-2 m) \vec{E}_{b b}, \vec{F}_{3}=(-2 m) \vec{E}_{e b}$, $\vec{F}_{4}=(m) \vec{E}_{b e}$. Then, it can be shown that the resultant force on a test mass $(m)$ due to a source mass $(M)$ is given by

$$
\vec{F}_{\text {resultant }}=\vec{F}_{1}+\vec{F}_{2}+\vec{F}_{3}+\vec{F}_{4}=-\frac{G M m}{r^{2}} \hat{r}=\vec{F}_{\text {Newtonian }}
$$

The resulting potential energies [6] of the test particle is given by the prescription $\vec{F}=-\vec{\nabla} U$, where $U$ being the potential energy, as $U_{1}=\frac{G M m}{r}, U_{2}=\frac{4 G M m}{r}, U_{3}=-\frac{3 G M m}{r}$ and $U_{4}=-\frac{3 G M m}{r}$ and their sum, $U_{\text {resultant }}=-\frac{G M m}{r}=$ $U_{\text {Newtonian. }}$.

Similarly, the potentials [6] $\Phi_{e e}=\frac{G M}{r}, \Phi_{b b}=-\frac{2 G M}{r}$, $\Phi_{e b}=\frac{3}{2} \frac{G M}{r}$ and $\Phi_{b e}=-\frac{3 G M}{r}$ do not add up to the Newtonian potential $\left(-\frac{G M}{r}\right)$, rather $\Phi_{\text {resultant }}=-\frac{5}{2} \frac{G M}{r}$. Hence the fields are color-sensitive, but the forces and potential energies are not, as it follows obviously.

Thus, Faruque [5] has clearly found a way to recover Newtonian force and potential energy [5] from four independent colorful fields in place of the single Newtonian field. One reason behind four fields instead of one is that physicists are trying to formulate quantum gravity theory using a spin 2 graviton field [7], but no satisfactory result has yet been found. We therefore are trying with a new approach with four fields. Here, the cross fields are 3/2 times the direct fields. To justify this, we can only say that the four fields are based on conjecture. The conjecture is justified by its giving the correct Newtonian gravitational force law and correct potential energy.

The four gravitoelectric fields have associated gravitomagnetic fields. So, there issues four set of Maxwellian equations. We have worked this out [8]. However, when we engage with quantum version of the theory, there would be four spin 1 bosons which will convey the gravitational interaction. This is a new avenue to visualize gravity field. Our result in [8] shows that effectively the fields acts like a spin 2 field.

This theory can be extended to a theory parallel to Gravitoelectromagnetism despite the fact that now one has four set of Maxwellian equation and each set involves a spin1 field. We call this fields spin1 gravitons when we set out to formulate a quantum theory of gravity. This theory will be called quantum gravitodynamics and it possesses four spin1 quantum bosons for transfer of the full gravitational force. In the next Section, we try to advance a little further in the direction of quantum gravity.

\section{Initial Concepts of the New Theory}

Electric mass ' $m$ ' and magnetic mass $(-2 m)$ can be thought of as two quantum states. The quantum operator [9, 10] for mass can be written as

$$
m_{o p} \rightarrow \frac{i \hbar}{c^{2}} \frac{\partial}{\partial t}
$$

Then for the electric mass ' $m$ ' states, the wave function is

$$
\psi_{e}=\exp \left(-\frac{i}{\hbar} m c^{2} t\right)
$$

And for the state of magnetic mass $(-2 m)$, the wave function is

$$
\psi_{b}=\exp \left(\frac{2 i}{\hbar} m c^{2} t\right)
$$

The operator which transforms (see figure 1c) $q(e)$ to $q(b)$ is simply

$$
\psi_{b e}=\exp \left(\frac{3 i}{\hbar} m c^{2} t\right)
$$

The four independent fields $\vec{E}_{e e}, \vec{E}_{b b}, \vec{E}_{e b}$ and $\vec{E}_{b e}$ can be considered as four color fields and in the language of quantum theory, we ascribe the symbols $q(e) \equiv m, q(b) \equiv$ $-2 m$, and the four fields by $q(e e), q(b b), q(e b)$ and $q(b e)$. The four fields $q(e e), q(b b), q(e b)$ and $q(b e)$ are distinct fields or spin 1 gravitons. They are exchanged when $q(e) \rightarrow$ $q(e)$ scattering or $q(b) \rightarrow q(b)$ scattering like interactions occur. $q(e)$ and $q(b)$ states are occupied by a single elementary particle simultaneously. However, when interactions occur the states $q(e)$ or $q(b)$ can act separately. Hence, we have four fundamental vertices when we consider Feynman diagrams [11] to be a means to envisage quantum gravity interactions. These are shown in figure 1.

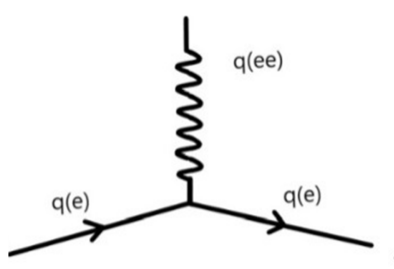

(a)

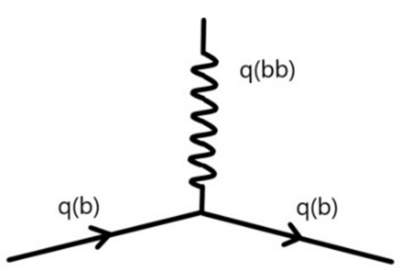

(b)

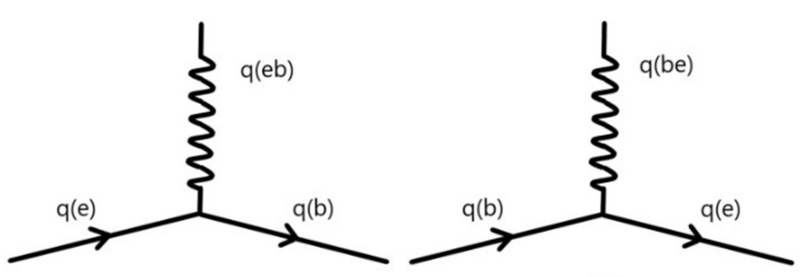

(c)

(d)

Figure 1. Fundamental vertices in quantum gravitodynamics

In figure 1(a) and 1(b), no flavor change occur, but in figure $1(\mathrm{c})$ and $1(\mathrm{~d}), q(e) \rightarrow q(b)$ and $q(b) \rightarrow q(e)$ type flavor changing occur.

Now, consider the interaction depicted in figure 1(a). Let us envisage potential scattering by a particle of electric mass $M$. The figure 1(a) would look like what is depicted in figure 2. 


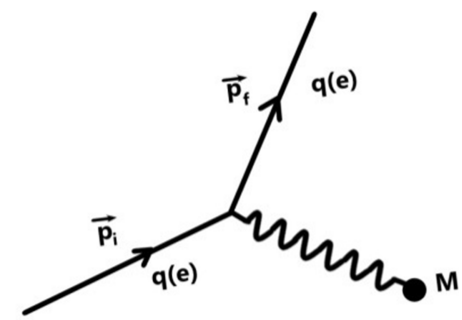

Figure 2. Potential scattering by a particle of mass $M$ as dictated by figure $1(a)$.

Let us think that $M$ is at the origin of coordinate system and $q(e)(\equiv m)$ is at $\vec{r}$. The potential energy in this situation is

$$
U_{1}(r)=\frac{G M m}{r}
$$

If the particle with $q(e)$ has initial momentum $\vec{p}_{i}$ and final momentum $\vec{p}_{f}$, its initial and final wave functions (time independent) are given by

$$
\psi_{i} \propto \exp \left(\frac{i \vec{p}_{i} \cdot \vec{r}}{\hbar}\right)
$$

and

$$
\psi_{f} \propto \exp \left(\frac{i \vec{p}_{f} \cdot \vec{r}}{\hbar}\right)
$$

Since, no flavor change occurs hence, the wave functions (12) and (13), need not contain any mass states. And, then, the amplitude for the transition (figure 1(a)) is

$$
A=\int \psi_{f}^{*} U_{1}(r) \psi_{i} d^{3} \vec{r} \propto G M m \int \exp \left\{\frac{i\left(\vec{p}_{i}-\vec{p}_{f}\right) \cdot \vec{r}}{\hbar}\right\} \frac{1}{r} d^{3} \vec{r}
$$

Performing the integral, we get

$$
A \propto \frac{G M m}{-|\vec{q}|^{2}},
$$

where, $\vec{q}=\vec{p}_{f}-\vec{p}_{i}$, is the momentum transferred from the scattered particle to the particle at origin. The amplitude for the scattering depicted in figure 1(b) has the same functional form except $G M m$ replaced by $(4 G M m)$. But for the scattering depicted in figures 1(c) and 1(d), we should consider the flavor change from $q(e) \rightarrow q(b)$ and from $q(b) \rightarrow q(e)$.

Since the state $q(e)$ changes to $q(b)$ in figure $1(c)$, the initial wave function is

$$
\psi_{i} \propto \exp \left(\frac{i \vec{p}_{i} \cdot \vec{r}}{\hbar}\right) \exp \left(\frac{-i}{\hbar} m c^{2} t\right)
$$

and the final state is

$$
\psi_{f} \propto \exp \left(\frac{i \vec{p}_{f} \cdot \vec{r}}{\hbar}\right) \exp \left(\frac{2 i}{\hbar} m c^{2} t\right)
$$

The interaction operator which transforms $\psi_{i} \rightarrow \psi_{f}$, can be written as

$$
V(r, t)=\frac{-3 G M m}{r} \exp \left(\frac{3 i}{\hbar} m c^{2} t\right)
$$

The total amplitude for the process of figure 1(c) is then

$$
\begin{gathered}
A \propto-3 G M m \int e^{\frac{-i \vec{p}_{f} \cdot \vec{r}}{\hbar}} e^{\frac{-2 i m c^{2} t}{\hbar}} e^{\frac{3 i m c^{2} t}{\hbar}} \frac{1}{r} e^{\frac{i \vec{p}_{i} \cdot \vec{r}}{\hbar}} e^{\frac{-i}{\hbar} m c^{2} t} d^{3} r \\
=-3 G M m \int \exp \left\{\frac{i\left(\vec{p}_{i}-\vec{p}_{f}\right)}{\hbar} \cdot \vec{r}\right\} \frac{1}{r} d^{3} r \\
=\frac{3 G M m}{|\vec{q}|^{2}} .
\end{gathered}
$$

For the scattering of the type depicted in figure 1(d), similar treatment is possible and warranted. Hence we have found a way to calculate transition amplitudes for basic Feynman diagrams that involve gravitational interaction.

\section{Scattering of Electrons from a Static Mass}

Now we calculate the Rutherford type scattering [11] of an electron at a fixed gravitational potential. Specifically, we consider the following diagram:

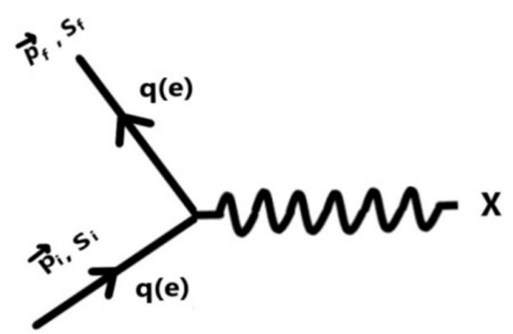

Figure 3. Scattering of an electron at an external potential $(X)$ to only gravitoelectric and lowest order.

Following Ref. [12], we write by replacing - ie by $i m_{e}$, where $m_{e}$ is the electron rest mass, the $S$-matrix for the scattering $S_{f i}$ as

$$
S_{f i}=i m_{e} \int d^{4} x \bar{\psi}_{f}(x) A_{0}(\vec{x}) \gamma^{0} \psi_{i}(x)(f \neq i)
$$

In the lowest order, $\psi_{i}(x)$ is approximately an incoming plane wave $\psi_{i}(x)$ of an electron with momentum $p_{i}$ and spin $S_{i}$ :

$$
\psi_{i}(x)=\sqrt{\frac{m_{e}}{E_{i} V}} u\left(p_{i}, s_{i}\right) e^{-i p_{i} \cdot x}
$$

where $u\left(p_{i}, s_{i}\right)$ is Dirac spinor of the incoming electron, $m_{e}$ is the mass of electron and $V$ denotes the volume of a box in which $\psi_{i}$ is normalized to probability 1 . Similarly, we can write

$$
\bar{\psi}_{f}(x)=\sqrt{\frac{m_{e}}{E_{f} V}} \bar{u}\left(p_{f}, s_{f}\right) e^{i p_{f} \cdot x}
$$

where $\bar{u}\left(p_{f}, s_{f}\right)$ is the adjoint Dirac spinor of the outgoing electron. The gravitational potential for $q(e) \rightarrow q(e)$ scattering by a static mass $M$ is given by (see section 1)

$$
A_{0}(x)=A_{0}(\vec{x})=\frac{G M}{|\vec{x}|}, \vec{A}(x)=0
$$

Hence equation (20) transforms to 


$$
S_{f i}=i \frac{G M m_{e}}{V} \sqrt{\frac{m_{e}^{2}}{E_{f} E_{i}}} \bar{u}\left(p_{f}, S_{f}\right) \gamma^{0} u\left(p_{i}, s_{i}\right) \int d^{4} x e^{i\left(p_{f}-p_{i}\right) \cdot x} \frac{1}{|\vec{x}|}
$$

The time integral of equation (24) can be reduced to $2 \pi \delta\left(E_{f}-E_{i}\right)$ as shown in [12].

The spatial part of integral is

$$
\int d^{3} x \frac{1}{|\vec{x}|} e^{-i \vec{q} \cdot \vec{x}}
$$

where, $\vec{q}=\vec{p}_{f}-\vec{p}_{i}$, the momentum transfer.

The integral in equation (25) can be evaluated using standard method. We find, thus,

$$
\int d^{3} x \frac{1}{|\vec{x}|} e^{-i \vec{q} \cdot \vec{x}}=\frac{4 \pi}{|\vec{q}|^{2}}
$$

Thus the $S$-matrix $S_{f i}$ becomes

$$
S_{f i}=i \frac{G M m_{e}}{V} \sqrt{\frac{m_{e}^{2}}{E_{f} E_{i}}} \bar{u}\left(p_{f}, S_{f}\right) \gamma^{0} u\left(p_{i}, s_{i}\right) \frac{4 \pi}{|\vec{q}|^{2}} 2 \pi \delta\left(E_{f}-E_{i}\right)
$$

Now, the number of final states $d N_{f}$ within the momentum range $d^{3} p_{f}$ is

$$
d N_{f}=V \frac{d^{3} p_{f}}{(2 \pi)^{3}}
$$

The transition probability per particle into these final states is given by [12]

$$
\begin{gathered}
d w=\left|S_{f i}\right|^{2} \frac{V d^{3} p_{f}}{(2 \pi)^{3}} \\
=\frac{M^{2}(4 \pi)^{2} \alpha_{G}^{2}}{E_{i} V} \frac{\left|\bar{u}\left(p_{f}, s_{f}\right) \gamma^{0} u\left(p_{i}, s_{i}\right)\right|^{2}}{|\vec{q}|^{4}} \frac{d^{3} p_{f}}{(2 \pi)^{3} E_{f}}\left(2 \pi \delta\left(E_{f}-E_{i}\right)\right)^{2},
\end{gathered}
$$

where we have used Planck units, $G=C=\hbar=1$, and the gravitational coupling constant [13] is employed, which is $\alpha_{G}=m_{e}^{2}$. The last factor in equation (29) is divergent, so to avoid that we assume the transition takes place within a finite interval of time $-\frac{T}{2} \leq t \leq \frac{T}{2}$. Then we can write

$$
2 \pi \delta\left(E_{f}-E_{i}\right) \rightarrow \int_{-T / 2}^{T / 2} d t \exp \left\{i\left(E_{f}-E_{I}\right) t\right\}=\frac{2 \sin \left(E_{f}-E_{i}\right) \frac{T}{2}}{E_{f}-E_{i}}(30)
$$

Hence,

$$
\left(2 \pi \delta\left(E_{f}-E_{i}\right)\right)^{2}=\frac{4 \sin ^{2}\left(E_{f}-E_{i}\right) \frac{T}{2}}{\left(E_{f}-E_{i}\right)^{2}} .
$$

The area under this function is

$$
\int_{-\infty}^{\infty} d E_{f} \frac{4 \sin ^{2}\left(E_{f}-E_{i}\right) \frac{T}{2}}{\left(E_{f}-E_{i}\right)^{2}}=2 \pi T
$$

To understand this result note that by replacing $\left(E_{f}-E_{i}\right)$ by $x$, the integral becomes $4 \sin ^{2}\left(\frac{x \frac{T}{2}}{x^{2}}\right)$. Now, the area under the curve can be approximated by a triangle with height $T^{2}$ and length of the basis $\frac{4 \pi}{T}$.

Therefore,

$$
\int_{-\infty}^{\infty} d x \frac{\sin ^{2}\left(x \frac{T}{2}\right)}{x^{2}} \approx \frac{1}{2} T^{2} \frac{4 \pi}{T}=2 \pi T
$$

When we increase $\mathrm{T}$ the shape of the function $4 \sin ^{2}\left(\frac{x \frac{T}{2}}{x^{2}}\right)$ approaches a $\delta$ function, the area under the curve assumes the value $2 \pi T$.

The square of the $\delta$-function can be written

$$
\begin{aligned}
\left(2 \pi \delta\left(E_{f}-E_{i}\right)\right)^{2} & =2 \pi T \delta(0) 2 \pi \delta\left(E_{f}-E_{i}\right) \\
& =2 \pi T \delta\left(E_{f}-E_{i}\right)
\end{aligned}
$$

This result can be understood in the following way:

$$
2 \pi \delta\left(E_{f}-E_{i}\right)=\lim _{T \rightarrow \infty} \int_{-T / 2}^{T / 2} d t \exp \left\{i\left(E_{f}-E_{I}\right) t\right\}
$$

For $E_{f}=E_{I}$, it follows that

$$
2 \pi \delta(0)=\lim _{T \rightarrow \infty} \int_{-\frac{T}{2}}^{\frac{T}{2}} d t=\lim _{T \rightarrow \infty} T
$$

Inserting Eq. (34) into Eq. (29), we get transition probability per particle per unit time with final states within momentum range $d^{3} p_{f}$ as,

$$
d R=\frac{d W}{T}=\frac{4 M^{2} \alpha_{G}^{2}}{E_{i} V} \frac{\left|\bar{u}\left(p_{f}, s_{f}\right) \gamma^{0} u\left(p_{i}, s_{i}\right)\right|^{2}}{|\vec{q}|^{4}} \frac{d^{3} p_{f}}{E_{f}} \delta\left(E_{f}-E_{i}\right)
$$

The scattering cross-section is the probability per particle per unit time divided by the incoming current of particles

$$
J_{\text {inc. }}^{a}=c \bar{\psi}_{i}(x) \gamma^{a} \psi_{i}(x)
$$

This form of the current of particles follows directly from the Dirac's theory of spin $\frac{1}{2}$ particles. Here, superscript $a$ in $J$ and $\gamma$ gives the component of the current vector in the direction of the velocity of the incoming particles,

$$
\vec{v}_{i}=\frac{\vec{p}_{i}}{E_{i}}
$$

It can be shown using appropriate spinors and with spin polarization along $Z$-axis that

$$
J_{\text {inc. }}^{a}=\frac{p_{i} c^{2}}{E_{i}} \frac{1}{V}
$$

With our choice of $c=1, E_{i}=m$ and $p_{i}=m v_{i}$, we get space part

$$
\left|\vec{J}_{\text {inc. }}\right|=\frac{\left|\vec{u}_{i}\right|}{V}
$$

Using equation (36) and equation (40), the differential cross-section becomes

$$
d \sigma=\frac{d R}{J_{i n c}}=\frac{4 M^{2} \alpha_{G}^{2}}{E_{i} V \frac{\left|\vec{u}_{i}\right|}{V}} \frac{\left|\bar{u}\left(p_{f}, S_{f}\right) \gamma^{0} u\left(p_{i}, s_{i}\right)\right|^{2}}{|\vec{q}|^{4}} \frac{\vec{p}_{f}^{2} d\left|\vec{p}_{f}\right|}{E_{f}} d \Omega_{f} \delta\left(E_{f}-E_{i}\right),
$$

where we have used momentum space volume element $d^{3} p_{f}=\vec{p}_{f}^{2} d\left|\vec{p}_{f}\right| d \Omega_{f}$. In practice, outgoing particles are 
counted taking into consideration only a small window of momentum $\Delta p_{f}$. Hence, the differential cross-section [14] per unit solid angle $d \Omega_{f}$ of the scattered particle follows as,

$$
\frac{d \sigma}{d \Omega_{f}}=\int_{\Delta p_{f}} \frac{4 M^{2} \alpha_{G}^{2}}{E_{i}\left|\vec{v}_{i}\right|} \frac{\left|\bar{u}\left(p_{f}, s_{f}\right) \gamma^{0} u\left(p_{i}, s_{i}\right)\right|^{2}}{|\vec{q}|^{4}} \frac{\vec{p}_{f}^{2} d\left|\vec{p}_{f}\right|}{E_{f}} \delta\left(E_{f}-E_{i}\right),
$$

which reduces to

$$
\frac{d \sigma}{d \Omega_{f}}=\frac{4 M^{2} \alpha_{G}^{2}}{|\vec{q}|^{4}}\left|\bar{u}\left(p_{f}, s_{f}\right) \gamma^{0} u\left(p_{i}, s_{i}\right)\right|^{2}
$$

In the non-relativistic limit, we expect to get a crosssection similar to Rutherford scattering cross-section [15]. To go to that limit, we note that in the non-relativistic limit, the four component spinors of the electron can be written as the states of $\sigma_{z}$, the Pauli matrix. Moreover, $\gamma^{0}$ reduces to a $(2 \times 2)$ form. Thus, in this limit we get

$$
\left|\bar{u}\left(p_{f}, s_{f}\right) \gamma^{0} u\left(p_{i}, s_{i}\right)\right|^{2}=1,
$$

And the cross-section reduces to

$$
\left.\frac{d \sigma}{d \Omega_{f}}\right|_{n . r . g}=\frac{4 M^{2} \alpha_{G}^{2}}{|\vec{q}|^{4}}
$$

This is gravitational analogue of Rutherford scattering cross-section.

In the case of Coulomb scattering by a static charge of $-Z e$, the corresponding formulae is

$$
\left.\frac{d \sigma}{d \Omega_{f}}\right|_{\text {Coulomb }}=\frac{4 Z^{2} \alpha^{2} m_{e}^{2}}{|\vec{q}|^{4}}
$$

If we take the scatterer as proton and restore usual units, Equation (44) becomes

$$
\left.\frac{d \sigma}{d \Omega_{f}}\right|_{n . r . g}=\frac{4 G^{2}\left(1836 m_{e}\right)^{2} m_{e}^{2}}{|\vec{q}|^{4}}
$$

and Equation (45) becomes

$$
\left.\frac{d \sigma}{d \Omega_{f}}\right|_{\text {Coulomb }}=\frac{4 e^{4} m_{e}^{2}}{|\vec{q}|^{4}}
$$

Hence the ratio of electrostatic to gravitostatic scattering cross-section of electron-proton scattering becomes

$$
\begin{gathered}
\frac{e^{4}}{G^{2}\left(1836 m_{e}\right)^{2}}=\frac{e^{4}}{(\hbar c)^{2}} \frac{\hbar c}{(1836)^{2} \frac{G m_{e}^{2}}{\hbar c} \cdot G} \\
=\frac{\alpha^{2}}{\alpha_{G}} \frac{\hbar c}{G} \frac{1}{(1836)^{2}} \\
\cong 1.7 \times 10^{17}
\end{gathered}
$$

where we have used the proper units for $\alpha$ and $\alpha_{G}$. In the language of high energy physics $\alpha=\frac{e^{2}}{\hbar c} \approx \frac{1}{137}$ and $\alpha_{G}=\frac{G m_{e}^{2}}{\hbar c}$.

\section{Discussion}

In summary, we have extended the elementary theory of
Faruque [5] to incorporate gravitational interactions between elementary particles according to the rules and mathematics of Dirac equation. We began our analysis with an excerpt of the theory of Faruque [5] in Section 1. This is classical physics and there are four independent color fields to convey the full gravitational force from the source of gravity to a test mass. The fields are colorful meaning that they do not add up to the Newtonian gravity field. Similarly for gravitational potential, they do not give the Newtonian potential when added. But the forces add up to the Newtonian force and also the potential energy add up to the Newtonian potential energy. So, four fields as shown in the section 1, suffice to convey gravity's influence.

In Section 2, we have discussed about the two states of a particle. We have shown that the gravitoelectric charge (or mass) and gravitomagnetic charge (or mass) can be considered as two states of the particle. Ordinarily, the particle occupies both of them. But when the question of interaction comes, the two states can get separated and take part in interaction. In this Section, we have shown the fundamental processes involving the two masses $q(e)$ and $q(b)$ in the fashion of Feynman. We have then considered the scattering of a particle off a static mass and found out the leading order terms of scattering amplitudes. We have worked out both $q(e) \rightarrow q(e)$ scattering and $q(e) \rightarrow q(b)$ type scattering amplitude. In the latter case, we had to take a course so that flavor change from $q(e) \rightarrow q(b)$ can be incorporated into calculation of amplitude. Our result is similar what we expect.

In Section 3, we take the process of electron scattering off a static mass $M$ and worked out in somewhat full detail the scattering cross-section. To do so, we have followed the procedure shown in [12]. In non-relativistic limit the formulas reduces to a formula like what we expect from Rutherford type scattering. In general, the $\frac{d \sigma}{d \Omega_{f}}$ is given by Equation (43). In the non-relativistic limit it is given by the simple formula of Equation (44). This result involve the gravitational coupling constant $\alpha_{G}$. Though it is very very small, interesting result came out when we take the nonrelativistic Rutherford scattering formula for Coulomb scattering of an electron off a static charge. This is given in Equation (45).

To have a feeling of the number involved we find the ratio of Coulomb scattering and gravitational scattering of an electron off a proton. We dressed up the formulae using the coupling constants $\alpha$ and $\alpha_{G}$, where $\alpha$ is the fine structure constant and $\alpha_{G}$ is the gravitational coupling constant.

To our surprise, the ratio is within the grasp of high energy physics, once a huge energy environment is considered. The ratio is only $\approx 10^{17}: 1$. Whereas gravitational coupling constant is $10^{42}$ times smaller than fine structure constant, the cross-sections are not so hugely separated in magnitude.

\section{Conclusion}

In conclusion, the new theory of gravity with four independent and color fields, gives interesting nonrelativistic and relativistic results for force, potential energy, 
scattering cross-sections. Feynman diagrams can also be easily handled with the new flavor changing operator $U_{f i}$. The ratio of Coulomb scattering cross-section to that of gravitation for scattering of electron off a static proton is also gives a good feeling since this is only $\approx 10^{17}: 1$. Thus we have moved a little forward to an as yet another theory of quantum gravity. A lot of work remains to do in future to complete the theory.

\section{Acknowledgements}

We acknowledge the support of NST under the Ministry of Science and Technology, Bangladesh. We also acknowledge the support of NLO Lab of the Department of Physics, Shahjalal University of Science and Technology, Sylhet, Bangladesh.

\section{References}

[1] Rindler. W (2001). Relativity: Special, General and Cosmological. $2^{\text {nd }}$ edition. Oxford University Press.

[2] McDonald. K. T. (1997). Am. J. Phys. 65, 7591.

[3] Mashhoon. B. (2000) Gravitational couplings of intrinsic spin. Class. Quantum Grav. 17 2399. https://doi.org/10.1088/0264$9381 / 17 / 12 / 312$.

[4] Mashhoon. B. (2005). Beyond Gravitomagnetism: critical speed in Gravitational Motion. International Journal of Modern physics D, V. 14, No. 12, pp 2025-2027. https://doi.org/10.1142/S0218271805008121.

[5] Faruque. S. B. (2018). A new approach to Gravity. arXiv: 1701. 06891v1 [physics.gen-ph].
[6] Aharonov, Y. Bohm, D. (1959) Phys. Rev. 11, 485. https://doi.org/10.1103/PhysRev.115.485.

[7] Regge, T. (1957). On the properties of spin 2 particles. Nuovo Cim 5, 325-336 https://doi.org/10.1007/BF02855242.

[8] Chakravorti, B. K. Islam, M. S. Faruque, S. B. (2020). A New Approach to Gravity Based on Multiple independent Fields. SUST J Sci Tech, Vol 30 (1), 25-31 ISSN 1682-895X.

[9] Da Costa, B. C. Borges, E. P. (2014). Generalized space and linear momentum operators in quantum mechanics. J. Math. Phys. 55, 062105. https://doi.org/10.1063/1.4884299.

[10] Griffiths, D. J. (1987). Introduction to Elementary particles. $2^{\text {nd }}$ edition, ISBN 0-471-60386-4. New York, Wiley, Print.

[11] Stöltzner, M. (2017). Feynman Diagrams as Models. Math Intelligencer 39, 46-54. https://doi.org/10.1007/s00283-0179716-z.

[12] Greiner, W. Reinhardt, J. (2009) Quantum Electrodynamics. $4^{\text {th }}$ edition, Springer-Verlag Berlin Heidelberg.

[13] Klinkhamer, F. R. (2011) Newton's Gravitational Coupling Constant from A Quantum of Area. Modern Physics Letters A. VOL. 26, NO. 17. pp 1301-1308 https://doi.org/10.1142/S0217732311035729.

[14] Huang, H. Liao, P. Chen, J. Wang, Y. (2014)"Absorption and Scattering Cross Section of Regular Black Holes", Journal of Gravity, vol. 2014, Article ID 231727. https://doi.org/10.1155/2014/231727.

[15] Bernal1, M A. Camacho, F J. Martinez, R (2013) Dimensional analysis and Rutherford scattering, Eur. J. Phys. 34 L5-L8. DOI: 10.1088/0143-0807/34/1/L5. 